\title{
The Effects of Chemical Fixation on the Cellular Nanostructure: A Correlative Study of Back-Scattered Interference Spectrometry Microscopy and TEM
}

Yue $\mathrm{Li}^{1}$, Luay Almassalha ${ }^{2}$, John Chandler ${ }^{3}$, Yolanda Cyrus ${ }^{3}$, Reiner Bleher ${ }^{4}$, Hariharan Subramanian ${ }^{3}$, Igal Szleifer ${ }^{3,5}$, Vadim Backman $^{3,5}$ *, Vinayak Dravid ${ }^{4,5^{*}}$

1. Applied Physics Program, Northwestern University

2. Interdisciplinary Biological Sciences Graduate Program, Northwestern University

3. Biomedical Engineering Department, Northwestern University

4. Materials Sciences and Engineering Department, Northwestern University

5. Chemistry of Life Processes Institute, Northwestern University

*. Corresponding Author(s)

When cryo-fixation is not applicable and chemical fixation is involved, there is a fair question to ask: how much cellular structure has changed due to fixation with respect to live cell's structure? Historically, intensive studies have been conducted using super-resolution techniques like EM and AFM [1]. Since most nanometer resolution techniques are invasive and time consuming, morphological changes are observed from limited numbers of different samples, thus a direct measurement over a physical quantity representing nanostructure before and after treatment is much needed. To meet this end, we employed the state-of-the-art Back-Scattered Interference Spectroscopic (BaSIS) [2] to monitor cellular structural changes in terms of the mass-density distribution during fixation with $20 \mathrm{~nm}$ sensitivity.

In this study, we employed BaSIS to analyze the changes in mass-density distribution induced by chemical fixation in one of the most commonly used protocols: individual cells flat-embedded in Spurr resin [3]. We started with Hela cell culture in a glass-bottom petri-dish (MatTek Corp.) and took two BaSIS measurements with cells in the incubator, one minute apart, to estimate the level of redistribution of mass-density due to natural cell dynamics (Fig1 (a) and (b)). In another area, we took a phase contrast image (Fig1 (c)) and BaSIS measurement of live cells (Fig1 (d)) and followed the protocol: 1. 2.5\% glutaraldehyde and $2 \%$ formaldehyde mixture fixation for 20 minutes (Fig1 (e)). 2. Stain $\mathrm{OsO}_{4}$ statining for 1h (Fig1 (f)). 3. Serial ethanol dehydration (Fig1 (g)). 4. Spurr's resin embedding and sectioning (Fig1 (h)). To quantify the structural changes, we aligned the BaSIS images based on highest correlation of the bright field images of the same cell taken at different stage and calculated statistical parameters including mean, coefficient of variance, skewness, kurtosis, and entropy for nucleus and cytoplasm respectively (Fig. 2 (a)). To quantify the pixel-to-pixel changes in mass-density distribution, we employed cross-correlation-coefficient in nucleus region and cytoplasm region for every step (Fig2 (b)).

From the bulk statistics, it seems that the standard protocol adequately preserves the cellular structure, as little change (less than 5\%) was observed. However, the lack of pixel-to-pixel cross-correlation (less than 0.3, while live cell measurements $1 \mathrm{~min}$ apart is more than 0.6) indicates neither the cell-dynamics state nor ultra-structure was preserved. Interestingly, when we compare the BaSIS value calculated from TEM structure with live cell BaSIS values, no significant correlation was obtained, while the correlation between calculated and measured values normally presents more than $80 \%$ correlation on the same sample. This lack of correlation suggests resin embedding is another source of possible structure change even if the cells are sufficiently fixed. 
Based on the quantitative analysis, we conclude that live cell imaging at nanoscale resolution is in great demand, and cryo-fixation is recommended whenever possible. For cases where neither live-cell nor cryo-fixation is feasible, the quantitative interpretation of cellular ultrastructure from cells fixed by any of the fixatives tested here should be made with great care. In the presentation, we will focus on the detailed experimental methods, as well as the future applications of this method, especially in broader range of in-vitro biological and biomedical research.

\section{References:}

[1] Murk, J. L. A. N. et al, Journal of microscopy, 212(1), 81-90, 2003.

[2] Subramanian et al, Optics letters, 34(4), 518-520, 2009.

[3] Reymond, O. L., \& Pickett-Heaps, J. D. Journal of microscopy, 130(1), 79-84, 1983.
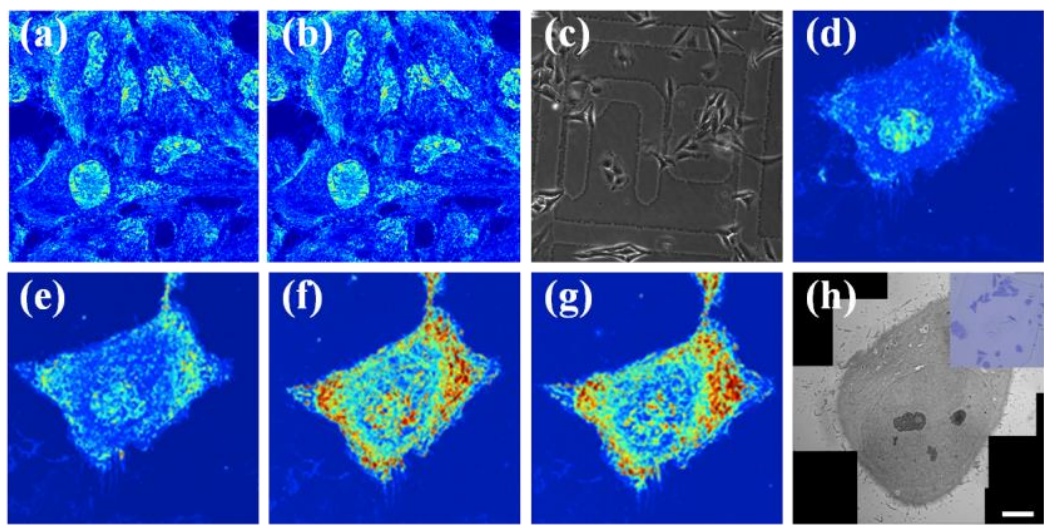

Figure 1. Monitoring fixation by BaSIS. For color coded images, redder indicates larger mass-density heterogeneity. (h) is the TEM image of the same cell in (d), scale bar: 5 um.

(a) Table 1 Statistics at Different Fixation Step

\begin{tabular}{|lllll|}
\hline Nucleus & Live & $\mathrm{GA}$ and FA & $\mathrm{OsO}_{4}$ & Serial ETOH \\
Mean & $0.048 \pm 0.005$ & $0.046 \pm 0.003$ & $0.078 \pm 0.008$ & $0.062 \pm 0.007$ \\
$\mathrm{CV}$ & $2.46 \pm 0.19$ & $2.51 \pm 0.16$ & $2.34 \pm 0.14$ & $2.22 \pm 0.09$ \\
Kurtosis & $3.15 \pm 0.34$ & $3.92 \pm 0.63$ & $3.89 \pm 1.25$ & $4.81 \pm 1.32$ \\
Skewness & $0.49 \pm 0.23$ & $0.85 \pm 0.15$ & $0.85 \pm 0.19$ & $1.11 \pm 0.24$ \\
Entropy & $11.96 \pm 0.27$ & $11.87 \pm 0.27$ & $12.50 \pm 0.41$ & $12.28 \pm 0.36$ \\
Absolute CCC & 1 & $0.28 \pm 0.09$ & $0.19 \pm 0.07$ & $0.07 \pm 0.36$ \\
Relative CCC & 1 & $0.28 \pm 0.09$ & $0.36 \pm 0.10$ & $0.25 \pm 0.08$ \\
Cytoplasm & Live & $\mathrm{GA}$ and FA & $\mathbf{O s O}_{4}$ & Serial ETOH \\
Mean & $0.030 \pm 0.006$ & $0.043 \pm 0.008$ & $0.083 \pm 0.018$ & $0.078 \pm 0.014$ \\
CV & $2.15 \pm 0.16$ & $1.98 \pm 0.004$ & $1.81 \pm 0.13$ & $1.76 \pm 0.01$ \\
Kurtosis & $5.46 \pm 1.98$ & $6.59 \pm 1.84$ & $6.71 \pm 2.27$ & $7.05 \pm 3.55$ \\
Skewness & $1.14 \pm 0.35$ & $1.39 \pm 0.34$ & $1.43 \pm 0.37$ & $1.41 \pm 0.39$ \\
Entropy & $11.66 \pm 0.31$ & $12.19 \pm 0.31$ & $13.16 \pm 0.37$ & $13.15 \pm 0.28$ \\
Absolute CCC & 1 & $0.35 \pm 0.07$ & $0.35 \pm 0.07$ & $0.31 \pm 0.06$ \\
Relative CCC & 1 & $0.35 \pm 0.07$ & $0.49 \pm 0.07$ & $0.55 \pm 0.06$ \\
\hline
\end{tabular}

(b)

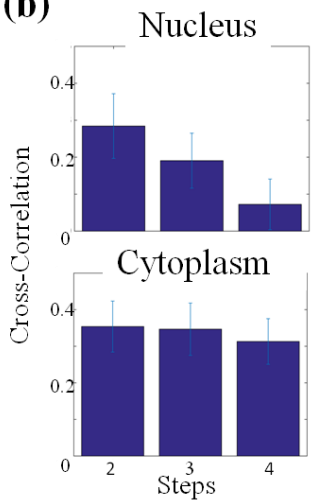

Figure 2. (a) Bulk statistics and (b) pixel-to-pixel correlation at each step for nucleus and cytoplasm respectively, calculated from over 100 Hela cells. 